\title{
Cardiopulmonary Exercise Test, mMRC and GAP score Can Predict 1-year Mortality in Patients with Idiopathic Pulmonary Fibrosis: A Prospective Study
}

\section{Yuan-Yang Cheng}

Department of Physical Medicine and Rehabilitation, Taichung Veterans General Hospital

Shih-Yi Lin

Center for Geriatrics and Gerontology, Taichung Veterans General Hospital

\section{Shin-Tsu Chang}

Department of Physical Medicine and Rehabilitation, Taichung Veterans General Hospital

\section{Chu-Hsing Lin}

Department of Computer Science, Tunghai University

Pin-Kuei Fu ( $\nabla$ yetquen@gmail.com )

Department of Critical Care Medicine, Taichung Veterans General Hospital

\section{Research Article}

Keywords: Idiopathic pulmonary fibrosis, 1-year mortality, Cardiopulmonary Exercise Test, GAP index, mMRC score

Posted Date: February 15th, 2021

DOI: https://doi.org/10.21203/rs.3.rs-192679/v1

License: (c) (i) This work is licensed under a Creative Commons Attribution 4.0 International License. Read Full License 


\section{Cardiopulmonary Exercise Test, mMRC and GAP score Can Predict 1-year Mortality in Patients with Idiopathic Pulmonary}

\section{Fibrosis: A Prospective Study}

Yuan-Yang Cheng ${ }^{1,2}$, Shih-Yi Lin ${ }^{2,3}$, Shin-Tsu Chang ${ }^{1,4,5,6}$, Chu-Hsing Lin ${ }^{7}$, Pin-Kuei

$\mathrm{Fu}^{7,8,9 *}$

${ }^{1}$ Department of Physical Medicine and Rehabilitation, Taichung Veterans General

Hospital, Taichung 40705, Taiwan

${ }^{2}$ School of Medicine, National Yang-Ming University, Taipei 11221, Taiwan

${ }^{3}$ Center for Geriatrics and Gerontology, Taichung Veterans General Hospital,

Taichung 40705, Taiwan

${ }^{4}$ Department of Physical Medicine and Rehabilitation, Tri-Service General Hospital,

Taipei 11490, Taiwan

${ }^{5}$ School of Medicine, National Defense Medical Center, Taipei 11400, Taiwan

${ }^{6}$ School of Medicine, Chung Shan Medical University, Taichung 40201, Taiwan

${ }^{7}$ Department of Computer Science, Tunghai University, Taichung 40705, Taiwan

${ }^{8}$ Department of Critical Care Medicine, Taichung Veterans General Hospital,

Taichung 40705, Taiwan

${ }^{9}$ College of Human Science and Social Innovation, Hungkuang University, Taichung 43302, Taiwan 
Corresponding author:

Dr. Pin-Kuei Fu

Division of Critical Care Medicine, Department of Internal Medicine, Taichung

Veterans General Hospital, Taichung, Taiwan

E-mail: yetquen@gmail.com 


\section{Abstract}

Idiopathic pulmonary fibrosis (IPF) is a rare lung disease with high mortality.

Finding an effective predictor of survival is therefore important for both clinicians and patients. In this prospective observational study, we evaluated the prognostic value of the parameters of cardiopulmonary exercise test (CPET), pulmonary function test (PFT), 6-min walk test (6MWT), and certain questionnaires on mortality in Asian patients with IPF. A total of 34 patients diagnosed with IPF were enrolled and followed up for 12 months, during which 6 patients died. The non-survivors had significantly higher minute ventilation to carbon dioxide output (VE/VCO2) slope, more oxygen desaturation during CPET and 6MWT, less heart rate recovery one minute after CPET, higher dead space, higher Gender-Age-Physiology (GAP) index and Modified Medical Research Council (mMRC) score. GAP index, mMRC score, VE/VCO2 slope, and end-tidal partial pressure of carbon dioxide (PETCO2) at maximal exercise demonstrated an area under curve (AUC) of $>0.7$, and the corresponding cut-off values were 4, 2, 35.1 and $3.6 \mathrm{kPa}$. Therefore, GAP index, mMRC scores, VE/VCO2 slope, and PETCO2 at maximal exercise could be important predictors for mortality in Asian patients with IPF.

Keywords: Idiopathic pulmonary fibrosis; 1-year mortality; Cardiopulmonary Exercise Test; GAP index; mMRC score 


\section{Introduction}

Idiopathic pulmonary fibrosis (IPF) is a progressive interstitial lung disease characterized by increased alveolar interstitial inflammation and cell proliferation, culminating in irreversible fibrotic changes. ${ }^{1,2}$ Compared with common chronic pulmonary diseases, the incidence of IPF is lower in East Asia and South America. ${ }^{3}$ However, its incidence and mortality rate are increasing steadily worldwide. ${ }^{4}$ Despite the disease course being highly variable, the median survival period is approximately 2-3 years from the time of diagnosis. ${ }^{5}$ Poor prognosis and the relatively short survival time have made it imperative to identify the appropriate predictors of survival for this patient population.

Many tools were developed for monitoring and evaluating the mortality risk of patients with IPF include pulmonary function test (PFT), ${ }^{6,7}$ cardiopulmonary exercise test $(\mathrm{CPET}),{ }^{8-14}$ and 6-min walk test $(6 \mathrm{MWT}){ }^{15-18}$. In IPF, a decrease in forced vital capacity (FVC) and diffusing capacity of the lungs for carbon monoxide (DLCO) are associated with increased mortality. ${ }^{19}$ Parameters in CPET test included maximum oxygen consumption, reduced ventilatory efficiency and exercise-induced hypoxaemia were all reported to have prognostic value in IPF. ${ }^{12}$ In $6 \mathrm{MWT}$, the distance walked in 6 min, ${ }^{20}$ decrease in the distance walked at 24 weeks,,${ }^{16}$ recovery of heart rate after 1 min of the test, ${ }^{17}$ and oxygen desaturation ${ }^{21}$ are associated with 
increased mortality in patients with IPF. Gender, age, and the two physiologic

variables in PFT "FVC" and "DLCO" have been recently integrated into the gender, age and pulmonary physiology (GAP) index, ${ }^{22}$ which can also precisely predict the survival rate in patients with IPF. $^{23}$

For monitoring IPF, the commonly used questionnaires that evaluate the overall health impact and the degree of breathlessness include Saint George's Respiratory Questionnaire (SGRQ) ${ }^{24-26}$, Short Form-36 (SF-36) ${ }^{27,28}$, and Modified Medical Research Council (mMRC) dyspnoea scale. ${ }^{29}$ Many of the mentioned tests predict the mortality of patients with IPF, but only some of them have been validated in Asians. ${ }^{7,23,26,29}$ The aim of the current study is to investigate the prognostic value of CPET, PFT, GAP index, SGRQ score, mMRC score, and SF-36 score for 1-year mortality in patients with IPF.

\section{Results}

\section{2-1. VE/VCP2 slope in CPET, mMRC and GAP score were higher in}

\section{non-survival group in patients with IPF}

In total, 34 patients with IPF were enrolled into to this study, and 6 patients died during the follow-up period within 1 year. Patients' data including age, gender, body mass index (BMI), CPET, PFT, 6MWT, GAP index, mMRC, SGRQ, and SF-36 scores were collected and compared between the survivors and non-survivors (Table 
1). We found no significant differences in age, gender and BMI between the groups.

In the data of CPET, higher VE/VCO2 slope, lower heart rate recovery one minute after exercise, higher minimal and maximal $\mathrm{Vd} / \mathrm{Vt}$, and more reduction in oxygen saturation was noted in the non-survivors. The more prominent oxygen desaturation was also observed in 6MWT of the non-survivors. In the data of PFT, no significant differences were noted between the two groups. Furthermore, lower GAP index and mMRC scores were also noted in the survivors.

\section{2-2. VE/VCO2 slope $>35.1$, PETCO2 $\leq 3.6 \mathrm{kPa}, \mathrm{mMRC} \geq 2$ and GAP score $\geq 4$ were} associated with 1-year mortality in patients with IPF

In order to find the predictors of mortality in our study participants, univariable cox regression analysis was done, and the data were shown in Table 2. Significant higher hazard ratio of mortality was observed in patients with lower PETCO2, higher VE/VCO2 slope, higher dead space, more desaturation in 6MWT, higher GAP index and higher mMRC score. However, further multivariable cox regression model failed to identify any parameters with significant $p$ value. ROC curve analysis was then performed to further identify the predictors excluding the effect of time to mortality. As shown in Table 3, we discovered that mMRC score, GAP index, PETCO2 and VE/VCO2 slope had an AUC > 0.7, and the cutoff value for them was 2, 4, $3.6 \mathrm{kPa}$, and 35.1, respectively. The curve of ROC analysis for the four parameters was 
displayed in Figure 1. The result of the De Long test was shown in Table 4, and no significant differences were established between the AUC of each two of the four parameters.

\section{Discussion}

In this preliminary study, we established the predictive value of CPET, GAP index and mMRC dyspnea score for mortality in an Asian cohort of patients with IPF. Although statistical significance could not be achieved in the multivariable Cox regression analysis, the four important parameters GAP index, mMRC dyspnea score, PETCO2 and VE/VCO2 slope in CPET, with a significantly large AUC in the ROC curve analysis were identified. These parameters can provide a reference to enable healthcare workers to manage Asian patients with IPF more effectively. To the best of our knowledge, this is the first study to confirm the mortality predictive role of CPET for Asian patients with IPF.

VE/VCO2 slope and PETCO2 have been considered to be two important prognostic factors in patients with interstitial lung disease and COPD, ${ }^{30}$ and both the two parameters were shown to be significant predictors of mortality in both the univariable cox regression analysis and ROC curve analysis of our study. In the influential consensus statement by European Association for Cardiovascular Prevention \& Rehabilitation and American Heart Association, the likelihood of 
secondary pulmonary hypertension increases if $\mathrm{VE} / \mathrm{VCO} 2$ slope $>45$ and resting PETCO $2<33 \mathrm{mmHg}$ with less than $3 \mathrm{mmHg}$ increase during CPET. ${ }^{30}$ Our study further added crucial supplement to the above statement, revealing the likelihood of mortality increases with VE/VCO2 slope $>35.1$ and exercise PETCO2 $<3.6 \mathrm{kPa}$ (27mmHg). PETCO2 is the partial pressure of carbon dioxide detected in the expired air during end expiration, reflecting the volume of alveolar dead space calculated by Bohr equation. ${ }^{31}$ Higher alveolar dead space, signifying worse ventilation/perfusion mismatch, is evidenced to contribute to mortality in critically ill patients. ${ }^{32,33}$ As shown in Table 1, our study also revealed higher resting and exercise dead space in the non-survivors. As pulmonary hypertension is a common consequence of IPF, the disease severity of pulmonary hypertension is evidenced to be proportional to PETCO2 at rest and exercise. ${ }^{34}$ Our study echoes the above literatures, and emphasizes the importance of mortality prediction in Asian IPF patients.

The indicator of ventilation efficiency, $\mathrm{VE} / \mathrm{VCO} 2$ slope, can never be overemphasized in the prediction of mortality in IPF patients. Past literatures had used the value of VE/VCO2 in the mortality prediction, with the cut-off value being $34{ }^{9}$ and $45^{35}$ at anaerobic threshold. A past study also showed that VE/VCO2 slope and $\mathrm{VO} 2 / \mathrm{kg}$ had the strongest correlation with the outcome of mortality. ${ }^{8}$ In our study, although no predictive role was established in VO2/kg, higher VE/VCO2 slope still 
demonstrated higher hazard ratio of mortality in cox regression analysis $(\mathrm{p}=0.029)$

and a large AUC> 0.7 in ROC analysis despite the $\mathrm{p}$ value was not significant

$(\mathrm{p}=0.108)$. The meaning of VE/VCO2 slope is straightforward, representing the

amount of ventilation volume needed per unit of carbon dioxide expelled. Therefore,

higher value of VE/VCO2 slope signifies ventilatory inefficiency, indicating higher

disease severity and higher likelihood of progression into pulmonary hypertension, ${ }^{36}$

and thus, higher likelihood of mortality.

Since 2012, the GAP index, which is derived from the patient's gender, age, FVC,

and DLCO, has been used for survival prediction. ${ }^{22}$ In addition, the index has been

successfully validated in Korean patients. ${ }^{37}$ However, the AUC for 1-year mortality

was only 0.619 for GAP stage in a recnelty nationwide cohort study in Korea. ${ }^{38}$ In our

study, although the AUCs of FVC and DLCO were not large enough to predict

mortality, the GAP index, with an AUC as high as 0.86, is a potential predictor of

survival. In contrast to the original version of GAP staging, which has two cut-off

values (i.e., $\leq 3$ and $\geq 6$ ) to define three stages of IPF, our result demonstrated only a

single cut-off value of $\geq 4$ between the two original values. Another simple

questionnaire, the mMRC, demonstrated the highest AUC of 0.87 in our study, which

is in line with the study by Nishiyama et al., who validated the predictive ability of

mMRC in patients with IPF. ${ }^{29}$ In our study, a cut-off value of $\geq 2$ had predictive 
mortality for patients with IPF, which happen to be the same for patients with COPD in an earlier study. ${ }^{39}$

Our study has several limitations. First, the follow-up period was short; thus, only a limited number of mortality cases were assessed in the one-year follow-up. If any of the data of the nonsurvivors were statistical outliers, the results of our study could be significantly affected. Second, the sample size was small. This was because of the relatively low prevalence of IPF in Taiwan (2.0-4.9 cases/100,000 persons). ${ }^{40}$ If more patients were to be included in our study, the results of the multivariable Cox regression and Kaplan-Meier curve analysis would have been more convincing. However, our study was a prospective single-center observational study of patients with newly diagnosed IPF. These preliminary results still hold value, especially for healthcare workers managing Asian patients with IPF.

\section{Methods}

\section{Study design}

This prospective observational cohort study was conducted in a tertiary medical centre in central Taiwan. Patients diagnosed as having IPF and who agreed to provide informed consent were enrolled from December 2018 to December 2020. The study protocol was reviewed and approved by the Institutional Review Board of Taichung 
Veterans General Hospital (IRB number, CE18325B; date of approval, Dec 18, 2018).

\section{Study population and setting}

In this study, a subgroup analysis of patients with a definite diagnosis of IPF according to the criteria of the American Thoracic Society, the European Respiratory Society, the Japanese Respiratory Society, and the Latin American Thoracic Association was conducted. ${ }^{41}$ The parameters included patient demographic data such as age, sex, height, and body weight. The index day was defined as the day on which the patient signed the inform consent form. The participants completed SGRQ, SF-36, and mMRC dyspnea questionnaires on the index day. Within 1 week of enrolment, the participants were administered PFT, 6MWT, and CPET. The patients were then followed up for 12 months to trace the incidence of mortality.

\section{Pulmonary function test}

In this study, PFT included two components. First, spirometry was conducted, which involves a period of quiet breathing, followed by expiration into the sensor as quickly and as long as possible after taking the deepest inspiration. FVC and FEV1 data were obtained from the results of the spirometry. Second, the difference in the partial pressure between the inspired and expired carbon monoxide was measured. After a deep inspiration, the participants had to hold their breath for 8-12 s. The DLCO value was obtained by measuring the subsequent air expiration. Both 
measurements were performed in accordance with the recommendations of the American Thoracic Society. ${ }^{42}$

\section{Cardiopulmonary exercise test}

We performed CPET by using an electromagnetically braked cycle ergometer.

All test procedures were performed in accordance with the guidelines of the American Heart Association and comprised 3-min rest, 3-min of unloaded pedalling, pedalling with the brake gradually applied in a ramp manner up to the maximal level tolerated by the patient, and unloaded pedalling for $3 \mathrm{~min} .{ }^{43}$ The ramp protocol to increase the workload during testing, in contrast to the conventional stepwise protocol, is characterized by the continuously gradual escalation of the work rate with even distribution within every moment of the exercise phase. CPET provided the following data: oxygen consumption in relation to body weight ( $\mathrm{VO} 2 / \mathrm{kg})$, functional aerobic impairment (FAI; ratio of the difference between the observed and the predicted peak oxygen consumption to the predicted peak oxygen consumption), percentage of peak oxygen consumption in relation to the heart rate (O2 pulse), end-tidal partial pressure of carbon dioxide (PETCO2) at maximal exercise, minute ventilation to carbon dioxide output (VE/VCO2) slope, heart rate recovery at $1 \mathrm{~min}$ after peak exercise (HR recovery), $\mathrm{O} 2$ desaturation, ratio of dead space to tidal volume at rest (maximal $\mathrm{Vd} / \mathrm{Vt}$ ), and maximal exercise (minimal $\mathrm{Vd} / \mathrm{Vt}$ ) and change in $\mathrm{Vd} / \mathrm{Vt}$ during the test. ${ }^{44}$ 
Furthermore, anaerobic threshold was determined by the V-slope method, ${ }^{45}$ which is the time when the rate the carbon dioxide production increases out of proportion to the rate of oxygen consumption.

\section{Six-Minute Walk Test}

6MWT was performed in accordance with the guidelines of the American Thoracic Society. ${ }^{46}$ The patients were instructed to walk as far as possible for 6 min in a corridor between the two orange traffic cones placed 30-m apart. Data on oxygen saturation and the distance walked in 6 min were obtained.

\section{Statistical analysis}

All the data were analysed using IBM SPSS Statistics 23.0 (Armonk, New York, USA) software. The Shapiro-Wilk test was used to examine variables with a normal distribution. For non-normally distributed data, the Mann-Whitney U and Fisher exact tests were used to determine the presence of any significant difference between survivors and nonsurvivors. The chi-square test was used to analyze discrete variables. Cox proportional regression analysis and receiver operating characteristic (ROC) curve analysis were performed for all the parameters measured. The parameters with areas under curve (AUCs) of $>0.7$ were identified, and the cut-off points were decided to maximize the sum of sensitivity and specificity values of the respective ROC curves. Furthermore, the De Long test was used to determine the differences between 
the AUCs of each two parameters. A p value of $<0.05$ was considered significant.

\section{References}

1 Lederer, D. J. \& Martinez, F. J. Idiopathic Pulmonary Fibrosis. N Engl J Med 378, 1811-1823, doi:10.1056/NEJMra1705751 (2018).

2 Wijsenbeek, M. \& Cottin, V. Spectrum of Fibrotic Lung Diseases. N Engl J Med 383, 958-968, doi:10.1056/NEJMra2005230 (2020).

3 Hutchinson, J., Fogarty, A., Hubbard, R. \& McKeever, T. Global incidence and mortality of idiopathic pulmonary fibrosis: a systematic review. Eur Respir J 46, 795-806, doi:10.1183/09031936.00185114 (2015).

4 Hutchinson, J. P., McKeever, T. M., Fogarty, A. W., Navaratnam, V. \& Hubbard, R. B. Increasing global mortality from idiopathic pulmonary fibrosis in the twenty-first century. Ann Am Thorac Soc 11, 1176-1185, doi:10.1513/AnnalsATS.201404-1450C (2014).

5 Kim, H. J., Perlman, D. \& Tomic, R. Natural history of idiopathic pulmonary fibrosis. Respir Med 109, 661-670, doi:10.1016/j.rmed.2015.02.002 (2015).

6 Collard, H. R. et al. Changes in clinical and physiologic variables predict survival in idiopathic pulmonary fibrosis. Am J Respir Crit Care Med 168, 538-542, doi:10.1164/rccm.200211-13110C (2003).

7 Nishiyama, O. et al. Prognostic value of forced expiratory volume in 1 second/forced vital capacity in idiopathic pulmonary fibrosis. Chron Respir Dis 13, 40-47, doi:10.1177/1479972315603714 (2016).

8 Triantafillidou, C. et al. The Role of Cardiopulmonary Exercise Test in IPF Prognosis. Pulmonary medicine 2013, 514817, doi:10.1155/2013/514817 (2013).

9 Vainshelboim, B., Oliveira, J., Fox, B. D. \& Kramer, M. R. The Prognostic Role of Ventilatory Inefficiency and Exercise Capacity in Idiopathic Pulmonary Fibrosis. Respiratory care 61, 1100-1109, doi:10.4187/respcare.04471 (2016).

10 Glaser, S. et al. Pulmonary hypertension in patients with idiopathic pulmonary fibrosis - the predictive value of exercise capacity and gas exchange efficiency. PloS one 8, e65643, doi:10.1371/journal.pone.0065643 (2013).

11 Molgat-Seon, Y., Schaeffer, M. R., Ryerson, C. J. \& Guenette, J. A. Cardiopulmonary Exercise Testing in Patients With Interstitial Lung Disease. Front Physiol 11, 832, doi:10.3389/fphys.2020.00832 (2020).

12 Barratt, S. L., Davis, R., Sharp, C. \& Pauling, J. D. The prognostic value of 
cardiopulmonary exercise testing in interstitial lung disease: a systematic review. ERJ Open Res 6, doi:10.1183/23120541.00027-2020 (2020).

13 Layton, A. M. et al. Cardiopulmonary exercise factors predict survival in patients with advanced interstitial lung disease referred for lung transplantation. Respir Med 126, 59-67, doi:10.1016/j.rmed.2017.03.022 (2017).

14 Phillips, D. B., Collins, S. E. \& Stickland, M. K. Measurement and Interpretation of Exercise Ventilatory Efficiency. Front Physiol 11, 659, doi:10.3389/fphys.2020.00659 (2020).

15 Lancaster, L. H. Utility of the six-minute walk test in patients with idiopathic pulmonary fibrosis. Multidiscip Respir Med 13, 45, doi:10.1186/s40248-018-0158-z (2018).

16 du Bois, R. M. et al. 6-minute walk distance is an independent predictor of mortality in patients with idiopathic pulmonary fibrosis. European Respiratory Journal 43, 1421-1429, doi:10.1183/09031936.00131813 (2014).

17 Swigris, J. J. et al. Heart rate recovery after 6-min walk test predicts survival in patients with idiopathic pulmonary fibrosis. Chest 136, 841-848, doi:10.1378/chest.09-0211 (2009).

18 Brown, A. W. \& Nathan, S. D. The Value and Application of the 6-Minute-Walk Test in Idiopathic Pulmonary Fibrosis. Ann Am Thorac Soc 15, 3-10, doi:10.1513/AnnalsATS.201703-244FR (2018).

19 Glaspole, l. et al. in C39. IPF: MORE ON DIAGNOSIS AND THERAPY A5004-A5004.

20 Mancuzo, E. V. \& Soares, M. R. Six-minute walk distance and survival time in patients with idiopathic pulmonary fibrosis in Brazil. 44, 267-272, doi:10.1590/s1806-37562018000000049 (2018).

21 Lettieri, C. J. et al. The distance-saturation product predicts mortality in idiopathic pulmonary fibrosis. Respir Med 100, 1734-1741, doi:10.1016/j.rmed.2006.02.004 (2006).

22 Ley, B. et al. A Multidimensional Index and Staging System for Idiopathic Pulmonary Fibrosis. Annals of Internal Medicine 156, 684-691, doi:10.7326/0003-4819-156-10-201205150-00004 (2012).

23 Lee, S. H. et al. Predicting survival of patients with idiopathic pulmonary fibrosis using GAP score: a nationwide cohort study. Respir Res 17, 131, doi:10.1186/s12931-016-0454-0 (2016).

24 Capparelli, I. et al. Translation to Spanish and Validation of the Specific Saint George's Questionnaire for Idiopathic Pulmonary Fibrosis. Arch Bronconeumol 54, 68-73, doi:10.1016/j.arbres.2017.09.004 (2018). 
25 O'Brien, E. C. et al. Disease Severity and Quality of Life in Patients With Idiopathic Pulmonary Fibrosis: A Cross-Sectional Analysis of the IPF-PRO Registry. Chest 157, 1188-1198, doi:10.1016/j.chest.2019.11.042 (2020).

26 Furukawa, T. et al. The St. George's Respiratory Questionnaire as a prognostic factor in IPF. Respiratory Research 18, 18, doi:10.1186/s12931-017-0503-3 (2017).

27 Verma, G., Marras, T., Chowdhury, N. \& Singer, L. Health-Related Quality of Life and 6 Min Walk Distance in Patients with Idiopathic Pulmonary Fibrosis. Canadian Respiratory Journal 18, 109635, doi:10.1155/2011/109635 (2011).

28 Zimmermann, C. S. et al. Comparison of two questionnaires which measure the health-related quality of life of idiopathic pulmonary fibrosis patients. Brazilian Journal of Medical and Biological Research 40, 179-187 (2007).

29 Nishiyama, O. et al. A simple assessment of dyspnoea as a prognostic indicator in idiopathic pulmonary fibrosis. European Respiratory Journal 36, 1067-1072, doi:10.1183/09031936.00152609 (2010).

30 Guazzi, M. et al. 2016 focused update: clinical recommendations for cardiopulmonary exercise testing data assessment in specific patient populations. Eur Heart J 39, 1144-1161, doi:10.1093/eurheartj/ehw180 (2018).

31 Waltuck, B. L. The Bohr equation. Anesthesiology 32, 472-473 (1970).

32 Bhalla, A. K., Belani, S., Leung, D., Newth, C. J. \& Khemani, R. G. Higher Dead Space Is Associated With Increased Mortality in Critically III Children. Crit Care Med 43, 2439-2445, doi:10.1097/ccm.0000000000001199 (2015).

33 Morales-Quinteros, L. et al. Estimated dead space fraction and the ventilatory ratio are associated with mortality in early ARDS. Ann Intensive Care 9, 128, doi:10.1186/s13613-019-0601-0 (2019).

34 Yasunobu, Y., Oudiz, R. J., Sun, X. G., Hansen, J. E. \& Wasserman, K. End-tidal PCO2 abnormality and exercise limitation in patients with primary pulmonary hypertension. Chest 127, 1637-1646, doi:10.1378/chest.127.5.1637 (2005).

35 Wallaert, B. et al. [Prognostic value of clinical exercise testing in idiopathic pulmonary fibrosis]. Rev Mal Respir 28, 290-296, doi:10.1016/j.rmr.2010.08.016 (2011).

36 Gläser, S. et al. Pulmonary hypertension in patients with idiopathic pulmonary fibrosis - the predictive value of exercise capacity and gas exchange efficiency. PloS one 8, e65643, doi:10.1371/journal.pone.0065643 (2013).

37 Kim, E. S. et al. Validation of the GAP score in Korean patients with idiopathic pulmonary fibrosis. Chest 147, 430-437, doi:10.1378/chest.14-0453 (2015). Lee, S. H. et al. Comparison of CPI and GAP models in patients with idiopathic 
pulmonary fibrosis: a nationwide cohort study. Sci Rep 8, 4784, doi:10.1038/s41598-018-23073-3 (2018).

39 Casanova, C. et al. Differential Effect of Modified Medical Research Council Dyspnea, COPD Assessment Test, and Clinical COPD Questionnaire for Symptoms Evaluation Within the New GOLD Staging and Mortality in COPD. Chest 148, 159-168, doi:10.1378/chest.14-2449 (2015).

40 Lai, C. C. et al. Idiopathic pulmonary fibrosis in Taiwan - a population-based study. Respir Med 106, 1566-1574, doi:10.1016/j.rmed.2012.07.012 (2012).

41 Raghu, G. Diagnosis of Idiopathic Pulmonary Fibrosis. An Official ATS/ERS/JRS/ALAT Clinical Practice Guideline. 198, e44-e68, doi:10.1164/rccm.201807-1255ST (2018).

42 Miller, M. R. et al. Standardisation of spirometry. Eur Respir J 26, 319-338, doi:10.1183/09031936.05.00034805 (2005).

43 Balady, G. J. et al. Clinician's Guide to cardiopulmonary exercise testing in adults: a scientific statement from the American Heart Association.

Circulation 122, 191-225, doi:10.1161/CIR.0b013e3181e52e69 (2010).

44 Bruce, R. A., Kusumi, F. \& Hosmer, D. Maximal oxygen intake and nomographic assessment of functional aerobic impairment in cardiovascular disease. American Heart Journal 85, 546-562, doi:https://doi.org/10.1016/0002-8703(73)90502-4 (1973).

45 Beaver, W. L., Wasserman, K. \& Whipp, B. J. A new method for detecting anaerobic threshold by gas exchange. J Appl Physiol (1985) 60, 2020-2027, doi:10.1152/jappl.1986.60.6.2020 (1986).

46 ATS statement: guidelines for the six-minute walk test. Am J Respir Crit Care Med 166, 111-117, doi:10.1164/ajrccm.166.1.at1102 (2002).

Acknowledgements : This research was funded by research grants from Taichung Veterans General Hospital (TCVGH-1094404C), and the Ministry of Science and Technology (Taiwan) (MOST 109-2410-H-075A-001-SSS)”.

Author contributions : Conceptualization, P.-K.F; Data curation, Y.-Y. C, S.-Y.L, and P.-K.F; Formal analysis, Y.-Y.C; Investigation, Y.-Y.C, S.-Y.L, S.-T.C, C.-H.L and P.-K.F; Methodology, Y.-Y.C and P.-K.F; Writing - original draft, Y.-Y.C and P.-K.F; Writing - review \& editing, Y-Y.-C, S-Y.L, S.-T.C, C.-H.L and P.-K.F. All authors read and approved the final manuscript

Competing Interests Statement : The authors declare no conflict of interest. 
Figure legends :

Figure 1. ROC curve analysis for the five parameters of mMRC score, GAP index, PETCO2, VE/VCO2 and DLCO. mMRC, Modified Medical Research Council; GAP index, gender, age, and the pulmonary physiology index; PETCO2, end-tidal partial pressure of carbon dioxide; $\mathrm{VE} / \mathrm{VCO} 2$, minute ventilation to carbon dioxide output; ROC, receiver operating characteristic.

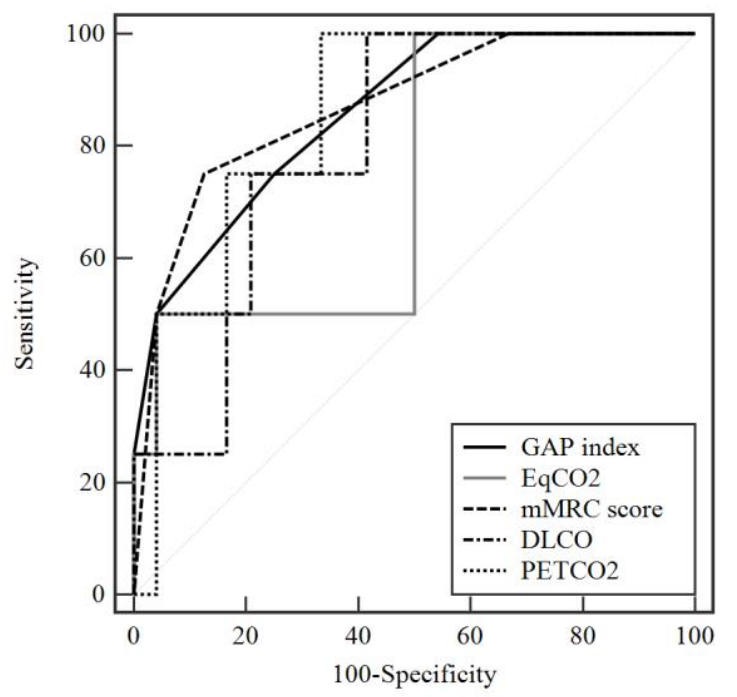


Tables :

Table 1. Parameters of patients with IPF stratified by survival status.

\begin{tabular}{|c|c|c|c|}
\hline Characteristics & Survivors $(n=28 ; 82 \%)$ & Non-survivors $(n=6 ; 18 \%)$ & $p$ value \\
\hline Age (years) & $68.5(26-81)$ & $69.0(67-86)$ & 0.459 \\
\hline Sex-Male, n (\%) & $20(71.4)$ & $5(83.3)$ & 0.928 \\
\hline BMI $\left(\mathrm{kg} / \mathrm{m}^{2}\right)$ & $24.3(15.5-34.0)$ & $25.2(16.6-27.5)$ & 0.644 \\
\hline \multicolumn{4}{|l|}{ CPET data } \\
\hline VO2/kg (ml/kg/min) & $14.0(8.5-20.9)$ & $12.7(8.7-18.0)$ & 0.490 \\
\hline FAI $(\%)$ & $32.0(15-59)$ & $32.5(24-58)$ & 0.732 \\
\hline PETCO2 (kPa) & $3.9(2.2-4.9)$ & $2.8(2.4-3.6)$ & 0.490 \\
\hline VE/VCO2 slope & $37.7(26.9-55.7)$ & $55.1(36.2-64.1)$ & $0.008^{* *}$ \\
\hline HR recovery (beat) & $11.1(1-26)$ & $2(0-10)$ & $0.020^{*}$ \\
\hline $\mathrm{SpO} 2$ reduction $(\%)$ & $5.0(0-16)$ & $10.0(4-17)$ & $0.044^{*}$ \\
\hline Maximal Vd/Vt & $31.0(16-56)$ & $47.0(25-53)$ & $0.047^{*}$ \\
\hline Minimal Vd/Vt & $27.0(7-51)$ & $43.0(16-51)$ & $0.037^{*}$ \\
\hline Change of $\mathrm{Vd} / \mathrm{Vt}$ & $4.0(1-14)$ & $4.0(1-9)$ & 0.672 \\
\hline \multicolumn{4}{|l|}{ Lung function test } \\
\hline FVC (liter) & $2.5(0.9-3.4)$ & $1.7(1.0-4.1)$ & 0.157 \\
\hline FVC (\% predicted) & $80.5(34-128)$ & $59.0(34-164)$ & 0.500 \\
\hline FEV1 (liter) & $2.0(0.9-2.8)$ & $1.5(1.0-2.6)$ & 0.200 \\
\hline FEV1 (\% predicted) & $81.5(42-132)$ & $65.0(46-217)$ & 0.419 \\
\hline FEV1/FVC (\%) & $82.5(55-98)$ & $90.0(62-99)$ & 0.112 \\
\hline DLCO (\% predicted) & $64.0(29-136)$ & $44.0(31-64)$ & 0.125 \\
\hline GAP index & $3.0(0-8)$ & $4.5(3-8)$ & $0.023^{*}$ \\
\hline \multicolumn{4}{|l|}{ 6MWT data } \\
\hline Resting saturation (\%) & $96.0(91.0-99.0)$ & $93.0(92.0-96.0)$ & 0.067 \\
\hline Exercise saturation (\%) & $90.0(79.0-97.0)$ & $77.0(74.0-86.0)$ & $0.001^{* *}$ \\
\hline SpO2 reduction (\%) & $6.0(0-17.0)$ & $16.0(6.0-22.0)$ & $0.018^{*}$ \\
\hline Distance (meters) & $414.5(138-554)$ & $426.0(38-464)$ & 0.797 \\
\hline mMRC score & $1.00(0-4)$ & $3(1-4)$ & $0.010^{*}$ \\
\hline \multicolumn{4}{|l|}{ SGRQ score } \\
\hline Symptoms score & $32.5(6.3-88.9)$ & $45.7(20.7-90.5)$ & 0.585 \\
\hline Activity score & $53.2(5.3-92.5)$ & $59.5(35.2-100)$ & 0.194 \\
\hline Impacts score & $35.1(3.1-85.6)$ & $29.0(5.3-91.7)$ & 0.842 \\
\hline Total score & $34.8(2.5-88.2)$ & $38.7(6.3-94.0)$ & 0.382 \\
\hline \multicolumn{4}{|l|}{ SF-36 score } \\
\hline Physical domain score & $53.8(12.5-93.8)$ & $40.0(24.4-81.3)$ & 0.473 \\
\hline Mental domain score & $47.0(12.4-95.5)$ & $48.8(25.8-93.8)$ & 0.920 \\
\hline Total score & $52.6(16.8-91.3)$ & $44.1(28.8-87.5)$ & 0.713 \\
\hline
\end{tabular}

CPET, cardiopulmonary exercise test; DLCO, diffusing capacity of the lungs for carbon monoxide; FAl, Functional aerobic impairment; FVC; forced vital capacity; FEV1; forced expiratory volume in $1 \mathrm{~s}$; GAP index, gender, age, and the pulmonary physiology index; HR, heart rate; IPF, idiopathic pulmonary fibrosis; mMRC, Modified Medical Research Council; PETCO2, end-tidal partial pressure of carbon dioxide; SF-36, Short Form-36; SGRQ, Saint George's Respiratory Questionnaire; Vd/Vt, ratio of dead space to tidal volume at rest; $\mathrm{VE} / \mathrm{VCO} 2$, minute ventilation to carbon dioxide output; $\mathrm{VO} 2 / \mathrm{kg}$, oxygen consumption in relation to body weight; $6 \mathrm{MWT}, 6$-min walk test. ${ }^{*} \mathrm{p}<0.05,{ }^{* *} \mathrm{p}<0.001$. 
Table 2. Univariate analysis of the mortality predictors in patients with IPF

\begin{tabular}{|c|c|c|}
\hline Variables & Univariate analysis & \\
\hline & HR $\quad(95 \% \mathrm{CI})$ & p value \\
\hline Age (year) & $1.08(0.96-1.21)$ & 0.214 \\
\hline Body mass index $(\mathrm{kg} / \mathrm{m} 2)$ & $2.00(0.20-19.91)$ & 0.554 \\
\hline \multicolumn{3}{|l|}{ CPET data } \\
\hline $\mathrm{VO} 2 / \mathrm{kg}(\mathrm{ml} / \mathrm{kg} / \mathrm{min})$ & $0.89(0.67-1.19)$ & 0.445 \\
\hline FAI $(\%)$ & $1.03(0.95-1.11)$ & 0.541 \\
\hline PETCO2 (kPa) & $0.11(0.01-0.85)$ & $0.034^{*}$ \\
\hline VE/VCO2 slope & $1.14(1.01-1.28)$ & $0.029^{*}$ \\
\hline HR recovery (beat) & $0.78(0.60-1.01)$ & 0.061 \\
\hline SpO2 reduction (\%) & $1.25(1.00-1.57)$ & 0.054 \\
\hline Maximal Vd/Vt & $1.13(1.01-1.27)$ & $0.040^{*}$ \\
\hline Minimal Vd/Vt & $1.12(1.00-1.26)$ & $0.049^{*}$ \\
\hline Change of $\mathrm{Vd} / \mathrm{Vt}$ & $0.92(0.62-1.37)$ & 0.697 \\
\hline \multicolumn{3}{|l|}{ PFT data } \\
\hline FVC (liter) & $0.05(0.15-1.69)$ & 0.263 \\
\hline FVC (\% predicted) & $1.00(0.97-1.03)$ & 0.963 \\
\hline FEV1 (liter) & $0.27(0.04-1.73)$ & 0.168 \\
\hline FEV1 (\% predicted) & $1.01(0.98-1.03)$ & 0.518 \\
\hline FEV1/FVC (\%) & $1.06(0.95-1.19)$ & 0.284 \\
\hline DLCO $(\%$ predicted $)$ & $0.96(0.90-1.02)$ & 0.142 \\
\hline GAP index & $1.83(1.06-3.16)$ & $0.029^{*}$ \\
\hline \multicolumn{3}{|l|}{ 6MWT data } \\
\hline Resting saturation (\%) & $0.58(0.32-1.06)$ & 0.076 \\
\hline Exercise saturation (\%) & $0.65(0.46-0.92)$ & $0.015^{*}$ \\
\hline SpO2 reduction (\%) & $1.42(1.06-1.89)$ & $0.018^{*}$ \\
\hline Distance (meters) & $1.00(0.99-1.00)$ & 0.356 \\
\hline mMRC score & $2.90(1.20-7.00)$ & $0.018^{*}$ \\
\hline \multicolumn{3}{|l|}{ SGRQ score } \\
\hline Symptoms score & $1.02(0.98-1.06)$ & 0.412 \\
\hline Activity score & $1.03(0.98-1.06)$ & 0.214 \\
\hline Impacts score & $1.00(0.97-1.03)$ & 0.945 \\
\hline Total score & $1.01(0.98-1.05)$ & 0.419 \\
\hline \multicolumn{3}{|l|}{ SF-36 score } \\
\hline Physical domain score & $0.99(0.94-1.03)$ & 0.489 \\
\hline Mental domain score & $1.00(0.96-1.04)$ & 0.891 \\
\hline Total score & $0.99(0.95-1.04)$ & 0.775 \\
\hline
\end{tabular}


$\mathrm{Cl}$, confidence interval; CPET, cardiopulmonary exercise test; DLCO, diffusing capacity of the lungs for carbon monoxide; FAI, functional aerobic impairment; FVC; forced vital capacity; FEV1; forced expiratory volume in 1 s; GAP index, gender, age, and the pulmonary physiology index; HR, hazard ratio; IPF, idiopathic pulmonary fibrosis; mMRC, Modified Medical Research Council; PETCO2, end-tidal partial pressure of carbon dioxide; PFT, pulmonary function test; SF-36, Short Form-36; SGRQ, Saint George's Respiratory Questionnaire; Vd/Vt, ratio of dead space to tidal volume at rest; VE/VCO2, minute ventilation to carbon dioxide output; VO2/kg, oxygen consumption in relation to body weight; $6 \mathrm{MWT}, 6$-min walk test. *p $<0.05$.

Table 3. ROC curve analysis of parameters with significant differences between the survivors and nonsurvivors

\begin{tabular}{|c|c|c|c|c|c|c|c|c|}
\hline Characteristics & AUC & $p$ value & $\begin{array}{c}\text { Cut-off } \\
\text { point }\end{array}$ & Sensitivity & Specificity & Accuracy & PPV & NPV \\
\hline mMRC score & $0.87^{* *}$ & $<0.01$ & $\geq 2$ & 75 & 87.5 & 85.7 & 50 & 95.5 \\
\hline GAP index & $0.86^{* *}$ & $<0.01$ & $\geq 4$ & 75 & 75 & 75 & 33.3 & 94.7 \\
\hline PETCO2 & $0.85^{* *}$ & $<0.01$ & $\leq 3.6$ & 100 & 66.7 & 71.4 & 33.3 & 100 \\
\hline VE/VCO2 slope & 0.74 & 0.108 & $>35.1$ & 100 & 50 & 57.1 & 25 & 100 \\
\hline
\end{tabular}

Table 4. Differences in the AUC between each of the two parameters of GAP index, PETCO2, VE/VCO2, and mMRC score on the De Long test

\begin{tabular}{ccc}
\hline Characteristics & Comparison parameters & $\boldsymbol{p}$ value \\
\hline \multirow{2}{*}{ GAP index } & VE/VCO2 slope & 0.561 \\
& mMRC score & 0.933 \\
& PETCO2 & 0.969 \\
\hline \multirow{2}{*}{ VE/VCO2 slope } & mMRC score & 0.331 \\
\hline mMRC score & PETCO2 & 0.413 \\
\hline
\end{tabular}

AUC, area under the curve; GAP index, gender, age, and the pulmonary physiology index; PETCO2, end-tidal partial pressure of carbon dioxide; VE/VCO2, minute ventilation to carbon dioxide output; mMRC, Modified Medical Research Council. 
Figures

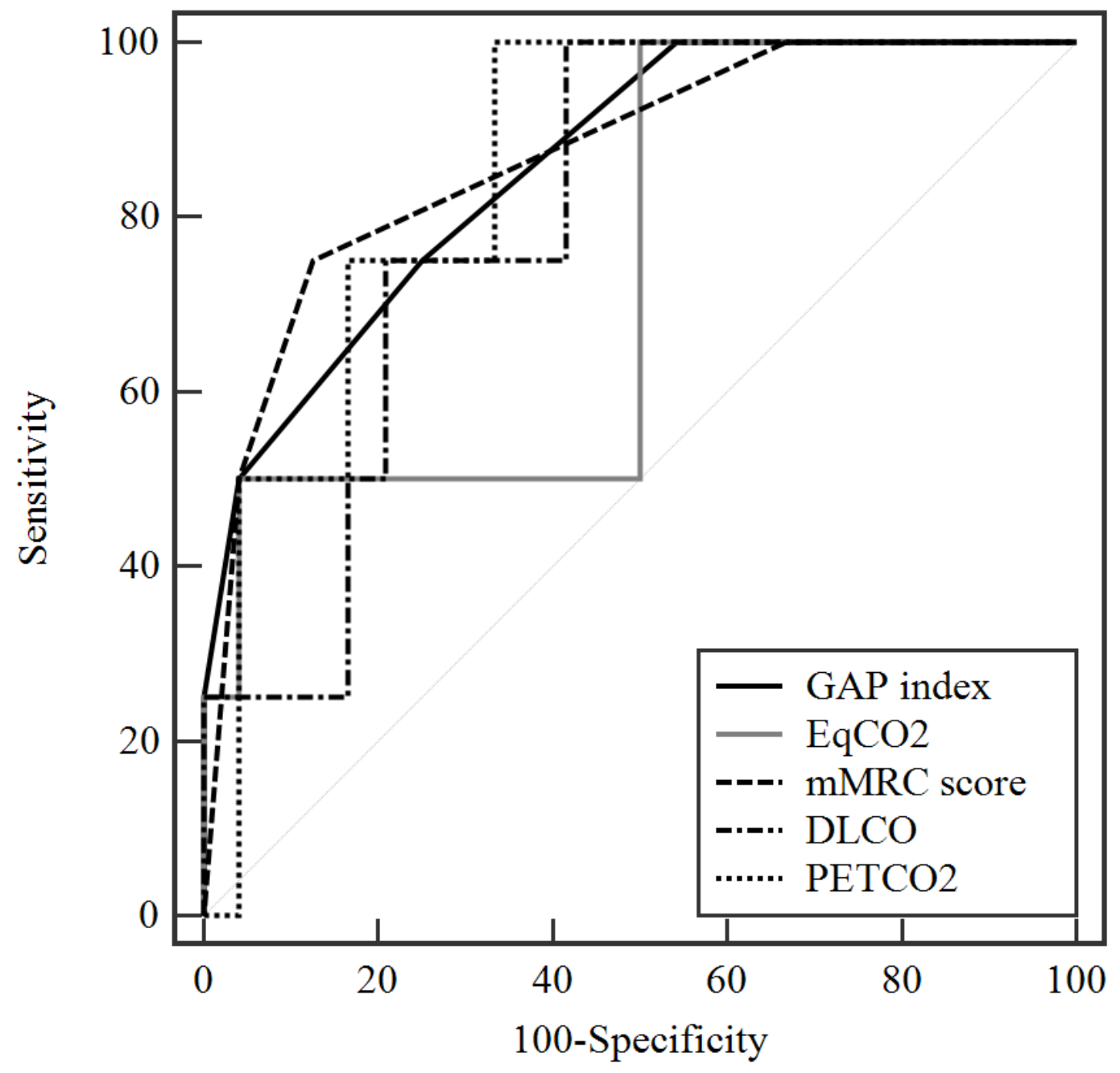

Figure 1

ROC curve analysis for the five parameters of mMRC score, GAP index, PETCO2, VE/VCO2 and DLCO. mMRC, Modified Medical Research Council; GAP index, gender, age, and the pulmonary physiology index; PETCO2, end-tidal partial pressure of carbon dioxide; VE/VCO2, minute ventilation to carbon dioxide output; ROC, receiver operating characteristic. 